\title{
Distances to Binary Pulsars and Implications for Tests of General Relativity
}

\author{
J. F. Bell \\ University of Manchester, NRAL, Jodrell Bank, Macclesfield, \\ Cheshire, SK119DLUK. email: jb@jb.man.ac.uk

\section{Bailes} \\ Physics Department, University of Melbourne, Parkville, \\ Victoria3052, Australia.email: mbailes@physics.unimelb.edu.au
}

\begin{abstract}
We propose a new way to measure accurate distances and transverse velocities for some nearby binary pulsars. In many cases the - distances will be more accurately determined than is possible by annual parallax, as the relative error decreases as $t^{-5 / 2}$. We also note that tests of the general relativistic prediction of orbital period decay of nearby relativistic binary pulsars will be limited to accuracies of a few percent. Nevertheless, PSR B1534+12 observations are consistent with general relativistic predictions if the proper-motion contribution to the orbital period derivative is accounted for.
\end{abstract}

\section{Distances and Velocities}

Weisberg (this proceedings) has summarised the methods by which distances to radio pulsars are commonly determined. Unfortunately, no pulsar has a distance estimate more accurate than $\sim 10 \%$, and for all but two, the errors are greater than $20 \%$.

Any acceleration of a pulsar along the line of sight will change the observed pulse period derivative $\dot{P}$. As Shklovskii (1970) pointed out, an apparent acceleration occurs when the proper motion is significant. The magnitude of this contribution is $\dot{P}_{(p m)} / P=v^{2} /(c d)$, where $P$ is the pulse period, $v$ is the transverse velocity, $d$ is the pulsar distance, and $c$ is the speed of light. For many millisecond pulsars, this effect is of similar magnitude to the intrinsic pulse period derivative (Camilo, Thorsett \& Kulkarni 1994).

This apparent acceleration also applies to orbital period derivatives, and the contribution is $\dot{P}_{b(p m)} / P_{b}=v^{2} /(c d)$, where $P_{b}$ is the orbital period (Damour $\&$ Taylor 1991). In fact, for many nearby millisecond pulsars, it is expected to completely dominate future observed orbital period derivatives. This means that $v^{2} / d$ can be obtained and, when combined with the measured proper motion $\mu=v / d$, the distance and transverse velocity can be easily separated. Hence, the proper-motion contribution to the pulse period derivative can also be evaluated, allowing the magnetic field strengths, characteristic ages and spin-down luminosities of binary millisecond pulsars to be determined. 
The amplitude and functional form of the residuals from a least-squares fit to the observed pulse arrival times, if one parameter is set to zero, is often called the "timing signal" for that parameter. For proper motion, the timing signal is often relatively large, with its amplitude increasing linearly with time. With continued measurement therefore, its relative error decreases as $t^{-1.5}$. The peak-to-peak amplitude $\Delta T_{(p m)}$ of the timing signal owing to the contribution of the proper motion to the observed orbital period derivative, is

$$
\Delta T_{p m}=\frac{a \sin i}{c} \frac{2 \pi}{P_{b}} \frac{v^{2}}{c d} t^{2}
$$

where $a$ is the semi-major axis of the pulsar's orbit and $i$ is the orbital inclination. The accuracy of distances obtained in this way are limited by the accuracy of the orbital period derivative measurements. Their accuracy, and therefore the accuracy of distances improve as $t^{-2.5}$. The fact that the relative error in both of these critical parameters decreases in such a rapid fashion demonstrates the power of this method for determining distance and transverse velocity.

Table 1 shows the predicted size of the timing signal $\Delta T_{p m}$ after 10 years of regular timing observations for a selection of binary millisecond pulsars. Where the proper motion was not available, the median transverse velocity for millisecond pulsars of $69 \mathrm{~km} \mathrm{~s}^{-1}$ was used. Also shown is the timing signal due to parallax (Ryba \& Taylor 1991), $\Delta T_{\pi}=r^{2} \cos ^{2} \theta /(2 c d)$, where $r$ is the radius of the Earth's orbit and $\theta$ is the angle between the line of sight to the pulsar and the Earth's orbital plane. After 10 years, the new method will provide better distance estimates than parallax measurements. This is possible because $\Delta T_{p m} \propto t^{2}$, while $\Delta T_{\pi}$ is constant. If an rms timing residual of $1.0 \mu \mathrm{s}$ could be obtained it would be possible to determine distances this way for several of the currently known binary millisecond pulsars. On average, for the pulsars listed in Table $1,2-3$ years of precise timing data have been recorded by various observers. So, to reap the rewards of this method a further $7-8$ years of precise timing will be required.

\section{Other Contributions to Period derivatives}

Other effects may contribute to observed orbital period derivatives (Damour \& Taylor 1991;Bell and Bailes 1996). These contributions are indistinguishable from the proper-motion contribution, so it is important to determine which of them are significant. Binary pulsars with either white dwarf or neutron star companions are very "clean", and few of these effects contribute. The only significant contributions to the orbital period derivatives of pulsar with neutron star or white dwarf companions are those due to acceleration in the Galactic potential $\dot{P}_{b(k z)}$, Galactic differential rotation $\dot{P}_{b(d r)}$, proper motion $\dot{P}_{b(p m)}$, and general relativity $\dot{P}_{b(g r)}$. Table 1 lists those contributions showing, that the proper-motion term will dominate for many of the binary millisecond pulsars.

For the nearby millisecond pulsar J0437-4715, the dominant uncertainty will be from $\dot{P}_{b(k z)}$ and is approximately $0.5 \%$ of $\dot{P}_{b(p m)}$, allowing the distance to be measured to an accuracy of better than $1 \%$. If the distance could be independently estimated with superior accuracy, it would be possible to determine the acceleration of the binary in the Galactic gravitational potential and 


\begin{tabular}{|l|cc|cccc|}
\hline \multicolumn{1}{|c|}{ Pulsar } & $\Delta T_{p m}$ & $\Delta T_{\pi}$ & \multicolumn{4}{c|}{ Contributions to $\dot{P}_{b} / P_{b(o b s)}\left(\times 10^{-19} \mathrm{~s}^{-1}\right)$} \\
Name & $(\mu s)$ & & $\dot{P}_{b(k z)} / P_{b}$ & $\dot{P}_{b(d r)} / P_{b}$ & $\dot{P}_{b(g r)} / P_{b}$ & $\dot{P}_{b(p m)} / P_{b}$ \\
\hline $\mathrm{J} 1713+0747^{1}$ & 0.3 & 1.0 & -0.89 & -0.29 & $\sim 0$ & 0.88 \\
$\mathrm{~B} 1855+09^{2}$ & 0.5 & 0.8 & -0.029 & -0.002 & -0.001 & 0.96 \\
$\mathrm{~J} 0613-0200^{3}$ & 1.5 & 0.4 & -0.33 & -0.67 & -0.25 & 2.4 \\
$\mathrm{~J} 2317+1439^{4}$ & 1.8 & 0.5 & -2.2 & 0.89 & -0.043 & 2.8 \\
$\mathrm{~J} 2145-0750^{5}$ & 2.2 & 2.4 & -1.3 & 0.033 & -0.009 & 2.1 \\
$\mathrm{~J} 0751+1807^{6}$ & 2.8 & 0.6 & -0.98 & -0.77 & -21.8 & 2.6 \\
$\mathrm{~J} 0034-0534^{5}$ & 3.4 & 1.2 & -2.8 & 0.17 & -0.15 & 5.3 \\
$\mathrm{~B} 1913+16^{7}$ & 3.8 & 0.1 & -0.064 & 5.4 & -845.3 & 0.74 \\
$\mathrm{~J} 2019+2425^{8}$ & 4.5 & 0.7 & -0.11 & 0.45 & $\sim 0$ & 12.6 \\
$\mathrm{~J} 1012+5307^{9}$ & 7.0 & 1.5 & -1.7 & 0.16 & -1.8 & 10.1 \\
$\mathrm{~J} 1022+1001^{10}$ & 13.5 & 2.0 & -1.8 & 0.048 & -0.010 & 8.7 \\
$\mathrm{~J} 1455-3330^{3}$ & 22.4 & 1.5 & -0.69 & -0.24 & $\sim 0$ & 73.1 \\
$\mathrm{~J} 0437-4715^{11}$ & 28.5 & 1.2 & -0.57 & 0.058 & -0.004 & 67.5 \\
$\mathrm{~B} 0655+64^{2}$ & 31.5 & 1.4 & -0.65 & -0.16 & -2.1 & 10.9 \\
$\mathrm{~B} 1534+12^{12}$ & 70.5 & 1.3 & -1.8 & -0.24 & -52.8 & 11.0 \\
\hline
\end{tabular}

Table 1. Predicted orbital period derivatives and timing signals. ${ }^{1}$ Camilo, Foster \& Wolszczan 1994, ${ }^{2}$ Taylor, Manchester \& Lyne 1993, ${ }^{3}$ Lorimer et al. 1995, ${ }^{4}$ Camilo, Nice \& Taylor $1993,{ }^{5}$ Bailes et al. 1994, ${ }^{6}$ Lundgren, Zepka \& Cordes 1995, ${ }^{7}$ Damour \& Taylor $1991,{ }^{8}$ Nice \& Taylor $1995,{ }^{9}$ Nicastro et al. $1995,{ }^{10}$ Camilo 1995, ${ }^{11}$ Bell et al. 1995, ${ }^{12}$ Arzoumanian 1995.

thereby constrain the distribution and composition of dark matter (Flynn \& Fuchs 1994). However, pulsars such as PSR J2317+1439 with large z-heights are probably more suitable as the contribution from the Galactic acceleration for such pulsars is comparable to the contribution from the proper motion.

\section{Implications for Tests of General Relativity}

The double neutron-star system PSR B1534+12 has been predicted to provide an even better general relativistic laboratory than PSR B1913+16 (Arzoumanian 1995). Unfortunately, the distance to this pulsar is known only to an accuracy $30 \%$ (Taylor \& Cordes 1993), and therefore the proper motion contribution to the orbital period derivative is uncertain by a similar amount. Recent measurements (Arzoumanian 1995) indicate that the predicted orbital period derivative due to gravitational wave emission $\dot{P}_{b(g r)}$ is $-1.924 \times 10^{-13}$, whereas the observed value $\dot{P}_{b(o b s)}$ is only $-1.5 \pm 0.3 \times 10^{-13}$. Using the dispersion-measure distance of $0.68 \pm 0.2 \mathrm{pc}$ and the measured proper motion, the contribution to the observed value from the proper motion is $\dot{P}_{b(p m)}=0.40 \pm 0.12 \times 10^{-13}$. Since $\dot{P}_{b(g r)}=$ $\dot{P}_{b(o b s)}-\dot{P}_{b(p m)}=-1.9 \pm 0.3 \times 10^{-13}$, the observed value is in excellent agreement with the general relativistic prediction. Unless the distance estimate can be 
improved, the orbital period decay due to the emission of gravitational waves cannot be verified to better than $\sim 5 \%$ in the PSR $1534+12$ system. This is a surprising result, which emphasises the importance of obtaining an accurate independent distance to this system.

\section{References}

Applegate, J. H. \& Shaham, J. 1994, ApJ, 436, 312.

Arzoumanian, Z. 1995. PhD thesis, Princeton University.

Bailes, M. et al. 1994, ApJ, 425, L41.

Bell, J. F. \& Bailes, M., 1996, ApJ, 456, L33.

Bell, J. F., et al. 1995, ApJ, 440, L81.

Camilo, F. 1995. PhD thesis, Princeton University.

Camilo, F., Foster, R. S., Wolszczan, A. 1994, ApJ, 437, L39.

Camilo, F., Nice, D. J., Taylor, J. H. 1993, ApJ, 412, L37.

Camilo, F., Thorsett, S. E., and Kulkarni, S. R. 1994, ApJ, 421, L15.

Damour, T. \& Taylor, J. H. 1991, ApJ, 366, 501.

Flynn, C. \& Fuchs, B. 1994, MNRAS, 270, 471.

Lorimer, D. R. et al. 1995, ApJ, 439, 933.

Lundgren, S. C., Zepka, A. F., and Cordes, J. M. 1995, ApJ, 453419.

Nicastro, L., et al. 1995, MNRAS, 273, L68.

Nice, D. J. \& Taylor, J. H. 1995, ApJ, 441, 429.

Ryba, M. F. \& Taylor, J. H. 1991, ApJ, 371, 739.

Shklovskii, I. S., 1970, Soviet Astron. MAJ, 13, 562.

Taylor, J. H. \& Cordes, J. M. 1993, ApJ, 411, 674.

Taylor, J. H., Manchester, R. N., Lyne, A. G. 1993, ApJS, 88, 529. 